\title{
Calculation of Texture Features for Polluted Leaves
}

\author{
S.K. Badugu ${ }^{1 *}$, R.K. Kontham ${ }^{2}$, V.K. Vakulabharanam ${ }^{3}$, B. Prajna ${ }^{4}$ \\ ${ }^{1 *}$ Dept. of CSSE, Andhra University College of Engineering (Autonomous), Visakhapatnam, India \\ ${ }^{2}$ Dept. of CSSE, Andhra University College of Engineering (Autonomous), Visakhapatnam, India \\ ${ }^{3}$ Dept. of CSE, JNTU Hyderabad, Hyderabad, India \\ ${ }^{4}$ Dept. of CSSE, Andhra University College of Engineering (Autonomous), Visakhapatnam, India \\ "Corresponding Author: suneel_it@hotmail.com, Tel.: +918919942456
}

Available online at: www.isroset.org

Received 08/Jan/2018, Revised 16/Jan/2018, Accepted 15/Feb/2018, Online 28/Feb/2018

\begin{abstract}
This paper discuss about polluted leaves texture features. Texture analysis such as segmentation and classification plays a vital role in computer vision and pattern recognition and is widely applied to many areas such as industrial automation , bio-medical image processing and remote sensing. Now a day's pollution is increasing day to day. classification of polluted leaves essential for some applications. To get the leaf based features image processing techniques are applied on the image of leaf. So texture features like mean, median ,skewness, kurtosis,GLCM and RMS of polluted leaves are published in this Article .Not only that Histogram of polluted leaves were published. Histograms of polluted leaves compared with data distribution measures like skewness and kurtosis.Manual polluted leafs identification task is time consuming process. Automatic detection of pollutant leaves is an active research topic now a days. Automatic polluted leaf identification will save time.In this paper histograms of nearly 30 polluted leafs of seven statistical measures were published.
\end{abstract}

\section{Keywords-Texture Features, Classification, Pollution, Histogram}

\section{INTRODUCTION}

In day to day life pollution is increasing in a huge manor in our Environment. Pollution because of so many reasons, release of so many gases like oxides from various Factories, Vehicles etc. These Pollutants causes dangerous for human health. The level of pollutants can be measured in various ways. One of the methods are by processing environmental leaves. So that by calculating texture features of leaves based on texture feature values anyone can automatically classify the leaves. The results of polluted/damaged/diseased leaf feature values are publishing in this article.

Rest of the paper is organized as follows, Section I contains the introduction of Polluted leaves, Section II contain the related work of Polluted/damaged leaves, Section III contain the some measures of methodology, Section IV contains the results and discussion, section $\mathrm{V}$ explain the conclusion and future work.

\section{RELATED WORK}

Environmental air pollution was measured by processing sick leaves/polluted leaves and also Relationship between number of sick leaves and air pollution was discussed by Anett C.Hansen and Harald K.Selte in [6].Effect of air pollution on chlorophyll content of leaves discussed by Sumitra giri in[1]. Sumitra[6] stated that reduction in the photosynthetic pigments of plant leaves growing in higher polluted leaves as compared to non or less polluted leaves.Rural area leaves and urbn area leaves were compared by Andrea Olbrich in [4] and also leaf size and stomata density were discussed and analyzed the result.Using light microscope leaf thickness ,height of palisade cells, diameter of the spongy cells , height and width of the upper epidermis cells, the thickness of the external wall of the upper epidermics cells,stomatal index were investigated at highly polluted sites and non polluted sites by University "Al.I.Cuza" Iasi in [6].Data distribution can classify the metals and non metals using Skewness ,Kurtosis Property as stated in [7].

Image J is a image processing and analysis in java tool.Some of the features of ImageJ is it runs everywhere.ImageJ is written in java,which allows it to run on Linux,Mac OS X and windows, in both 32-bit and 64 bit modes.ImageJ is a 
OpenSource that means ImageJ and its Java source code are freely available in the public domain ,No License is required.ImageJ has a large and knowledgeable worldwide user community .More than 1700 users and developers subscribe to the ImageJ mailing list.Using macros ImageJ automate tasks and create custom tools.Generate macro code using the command recorder and debug it using the macro debugger.More than 300 macros are available on the ImageJ Website.ImageJ has Plugins.Extended ImageJ by developing plugins using ImageJ's built in text editor and java compiler.More than 500 plugins are available.Use ImageJ as a image processing toolkit(class library) to develop applets ,servelts or applications.ImageJ is the worlds fastest pure Java image processing program.It can filter a 2048x2048 image in 0.1 seconds $(*)$.That's 40 million pixel per second.ImageJ has several data types and File formats.

\section{Methodology}

For Processing images so many softwares /tools are available.Python is an interpreted high level programming language for general purpose programming.Python has some libraries like scikit-learn,wradlib etc to process image data.Matlab also very useful for processing images.And $\mathrm{R}$ ,RapidMiner ImageJ are some of the tools/Softwares which deals with images.

Image J is Image Proscessing and analysis in java .Features of ImageJ s are given below

1)Runs Everywhere

2)Open Source

3)User Community

4)Macros

5)Plugins

6)Toolkit

7)Speed

8)Data Types

9)File formats

10)Image Display
11)Selections

12)Image Enhancement

13)Geometric operations.

14)analysis

15)editing

16)Color Processing

17)stacks

Processing of images can be done through image processing techniques. Operations can be performed on images in order to get some useful information from those images. Images can be processed, segmented, enhanced and so on using different image processing techniques. Images available in color as well as gray scale format. Texture is one of the features that can be helpful to segment images into regions of interest.Textures are one of the important features in computer vision for many applications[2].And that reigons can be classified according to results.Texture can be seen in many images from multispectral remote sensed data to microscopic photography .Each Texture analysis method characterizes image texture in terms of the features it extracts from the image[3].Structure analysis schemes can be categorized in to statistical,structural and spectral approaches.Three primary issues in texture analysis, such as texture classification,texture segmentation and shape recovery from texture[3].In texture classification, the problem is identifying the given texture region from a given set of texture classes.In texture classification class label of a single homogeneous region is determined using distinguish features derived from the region. In texture segmentation boundaries are determined between various textured regions in an image.Image segmention is essentially the process of dividing an image into small pieces or objects that have a common attribute ,e.g .,color ,brightness ,texture ,boundary continuity and so on[3]. Texture features includes Mean, Median, Skewness, Kurtosis,RMS(ROOT MEAN SQUARE) and GLCM(GRAY LEVEL CO-OCCURANCE MATRIX).Texture Gives us information about the spatial arrangement of the colors or intensities in an image.The Classification problem is basically the problem of identifying an observed textured sample as one of several possible texture classes by a reliable but computationally attractive texture classifier.Initially texture analysis was based on the first order second order statistics of textures.According to Choras[] texture is a powerful regional descriptor that helps in retrieval process.Texture, On its own does not have capability of finding similar images, but it can be used to 
classify textured images from non-textured ones to make the retrieval more effective. Texture features can be extracted by using various methods.Gray-level occurance matrices(GLCMs), Gabor Filter,and Local binary pattern(LBP) are examples of popular methods to extract textures features.Other methods to get texture features is using fractals[13].Fractals in texture classification have been discussed in [14].Fractal application in image retrieval has been applied by Min et all[15].There is a fractal measure called lacunarity,which is a measure of non-homogeineity of the data[16].lacunarity measures lumpiness of the data.lacunarity defined in term of the ratio of the variance over the mean value of the function .

Local Binary Pattern :Very Simple ,but useful texture measure is the local binary partition measure.For each pixel $p$ in the image ,The eight neighbouts are examined to see if their intensity is greater that that of p.The results from eight neighbours are used to construct an eight-digit binary number b1,b2,b3,b4,b5,b6,b7,b8 where bi=0 if the intensity of the ith neighbour is less than or equal to that of $p$ and 1 otherwise.A histogram of these numbers is used to represent of the image.

Mean:Mean represents the mean value of the gray levels. The values of the mean is high when the sum of gray level is high.

$$
\mu=\frac{1}{M N} \sum_{I=1}^{M} \sum_{J=1}^{N} P_{I J}
$$

Mean $=($ values of the pixels in the image $) /($ Number of pixels in the image)

\section{Standard Deviation:}

The standard deviation $\sigma$ represents the amount of variation or the dispersion data set can be represented by standard deviation ,the low standard deviation refer to the value of data which tend to be close to the mean, while a high standard deviation indicates that the data set is dispersion over a wider range of the data values. The standard deviation is calculated as

$\sigma=\sqrt{\frac{1}{M N}} \sum_{I=1}^{M} \sum_{J=1}^{N}\left(P_{I J}-\mu\right)^{2}$

Measures of Skewness and Kurtosis:

Skewness is a measure of symmetry or more precisely the lack of symmetry.A distribution, or data set is symmetric if it looks the same to the left and right of the center point. Skewness , which characterizes the asymmetry of a distribution is classically defined as the normalized third moment.A fundamental task in many statistical analysis is to characterize the location and variability of a data set.For univariate data $\mathrm{y} 1$ and $\mathrm{y} 2 \ldots . . \mathrm{yn}$ the formula for skewness is
SKEWNESS $=\frac{\sum_{I=1}^{N}\left(Y_{i}-\bar{Y}\right)^{3}}{(N-1) S^{3}}$

Where $\bar{Y}$ is the mean, $\mathrm{S}$ is the standard deviation and $\mathrm{N}$ is the number of the data points .

The skewness for a Normal distribution is zero,and any symmetric data should have a skewness near zero.Negative values for the skewness indicate data that are skewed left and positive values for the skewness indicate data that are skewed right.By skewed left, we mean that the left tail is long relative to the right tail.Similarity right means that the right tail is long relative to the left tail some measurements have a lower bound and are skewed right Kurtosis is a measure of whether the data are peaked or flat relative to normal distribution .That is data sets with high kurtosis tend to have a distinct peak near the mean, decline rather rapidly and have heavy tails.Data sets with low kurtosis tend to have a flat top near the mean rather than a sharp peak.Histogram is an effective graphical technique for showing both the skewness and kurtosis of data sets[11].

If skewness $>0$ Then distribution is right skewed.That means mean $>$ median.

Most values are concentrated on left of the mean ,with extreme values of the right.

If Skewness $<0$ Then distribution is left skewed.That means mean $<$ median.

Most values are concentrated on the right of the mean with extreme values of the left.

If Skewness $=0$ it means

Mean=median

The distribution is symmetrical around the mean[11].

Graylevel Histogram Based Features:

In histogram-based approach ,the analysis of texture is done on the basis of the intensity values of an entire image or part of image.These intensity values provide statistical information. The statistical methods can be used to check the gray values.Features derived from gray level histogram based approach include different moments and are described below. Number of pixels:It can be further classified as first-order that deals with only one pixel.This estimates properties of values of each pixel.There can be two pixels and higher order pixels, which estimates properties of two or more pixel values[17].There are statistical techniques like mean,standard deviation,variance,skewness etc.Co occuranc matrix is a successful method since it provides pixel values at different locations that are relative to each other. 
Moment:First moment(mean).second moment,third central moment and fourth central moment based classification[17].

Co-Occurrencthe Matrix:A co-occurance matrix is a two dimensional array $\mathrm{C}$ in which both the rows and th columns represent a set of possible image values V.Co-Occurance matrices capture properties of a texture,but they are not directly useful for further analysis, such as comparing two textures.Instead,numeric features are computed from the cooccurance matrix that can be used to represent the texture more compactly.The following are the Standard co-occuranc e matrix.Homogeneity represents smoothness degree.

Energy $=\sum_{\mathrm{i}} \sum_{\mathrm{j}} \mathrm{N}_{\mathrm{d}}^{2}(\mathrm{i}, \mathrm{j})$

Entropy $=-\sum_{i} \sum_{j} N_{d}(i, j) \log _{2} N_{d}(i, j)$

Homogeneity $=\sum_{i} \sum_{j} \frac{N_{d}(i, j)}{1+/ i-j /}$

contrast $=\sum_{i} \sum_{j}(i-i)^{2} N_{d}(i, j)$

Correlation $=\frac{\sum_{i} \sum_{j}\left(i-\mu_{i}\right)\left(j-\mu_{j) N} d(i, j)\right.}{\sigma_{i \sigma_{j}}}$

Where $\mu_{i, \mu_{j}}$ are the means and $\sigma_{i} \sigma_{j}$ are the standard deviations of the row and column.

Sums $N_{d}(i)$ and $N_{d}(j)$ defined by

$N_{d}(j)=\sum_{i} N_{d}(i, j)$

$$
N_{d}(i)=\sum_{j} N_{d}(i, j)
$$

Root Mean Square Value:

RMS value means square root of mean value

Images of various polluted leaves are acquired using a digital camera.Then image processing techniques are applied to the acquired images to extract useful features that are necessary for further analysis. Approximately 20 to 30 images have taken using digital phone camera

\section{Algorithm:}

Read the RGB image Using imread function in MATLAB and

Calcualte Histogram,Mean,Median,Skewness,Kurtosis ,RMS,GLCM

Repeat the step until all images are over.

Compare mean with median

This is the code for calculating histogram using python from matplotlib.mlab import PCA

import cv2

from PIL import Image

import numpy as np

img $=$

cv2.imread('E:Isecuritylcc.jpg',cv2.IMREAD_GRAYSCALE)

print(type(img))

\# calculate frequency of pixels in range 0-255

histg $=$ cv2.calcHist $([\mathrm{img}],[0]$, None, $[256],[0,256])$

\# find frequency of pixels in range 0-255

color $=\left(' b^{\prime},{ }^{\prime} g ', ' r '\right)$

for $\mathrm{i}, \mathrm{col}$ in enumerate(color):

histr $=$ cv2.calcHist $([\mathrm{img}],[0]$, None, $[256],[0,256])$

\# show the plotting graph of an image

hist,bins $=$ np.histogram $($ img, $256,[0,256])$

plt.show()

plt.plot(histr)

plt.show()

\section{Code for calculating GLCM Using MATLAB}

p1=imread('E:Isecuritylooo.jpg');

$\mathrm{p} 2=\operatorname{rgb} 2 \operatorname{gray}(\mathrm{p} 1)$;

glcm=graycomatrix(p2,'offset',[0 1],'Symmetric',true);

stats $=$ graycoprops $($ glcm,$\{$ 'all' $\})$

$\mathrm{X}=\operatorname{reshape}(\mathrm{p} 1, \operatorname{size}(\mathrm{p} 1,1) * \operatorname{size}(\mathrm{p} 1,2), 3)$;

Code For Calculating mean,median,kurtosis,skewness using MATLAB

p1=imread('E:Isecurityla.jpg');

$[\mathrm{m}, \mathrm{n}]=\operatorname{size}(\mathrm{p} 1)$

$\mathrm{p} 1=$ double(p1);

$\mathrm{m}=\operatorname{mean}(\mathrm{p} 1)$;

sprintf('MeanOf the Image\%3.8f \n\n',m);

md=median(p1);

sprintf('MedianOftheImage $=\% 3.8 \mathrm{f} \backslash n \backslash n ', \mathrm{~m}$;

$\mathrm{k} 1=\operatorname{kurtosis}(\mathrm{p} 1)$;

sprintf('kurtosisOftheImage $=$

\%3.8f $\backslash n \backslash n ', k 1)$;

sk=skewness $(\mathrm{p} 1)$;

sprintf('skewness Of the Image \%3.8fınไn',sk);

\section{Results and Discussion}

By using above methodologies using python,Matlab and ImageJ softwares statistical measures of Polluted leaves calculated and Published the results.

import numpy as np

import matplotlib.pyplot as plt 


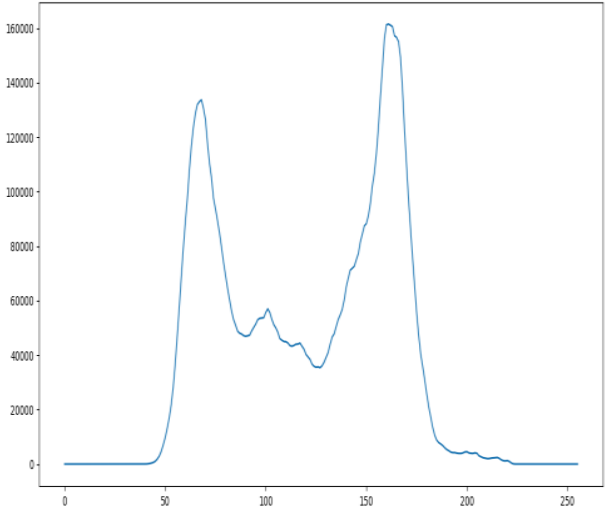

Figure 1 Histogram

Mean: 121.0434 ;Median: min-53,max-178 ; skewness:min0.5184 , max-3.8541; Kurtosis

:min-1.4799

,Max:3.6612;Standard deviation: 128.49916451;rms: 128.49916451;Correlation:0.9911,Energy:0.2086,Homogene ity:0.9841

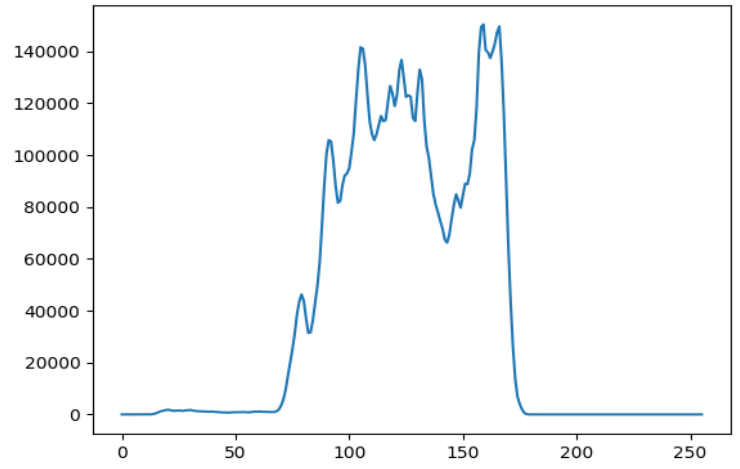

Figure 2.Histogram

Mean: $\quad$ 137.9225; $\quad$ Median:117<177;skewness:$1.3236<0.7825$;kurtosis: $1.4746<4.2898$; standard deviation: 17.65411786;rms: 139.04778278; Contrast: 0.0193; Correlation: 0.9705; Energy: 0.4781; Homogeneity: 0.9903;

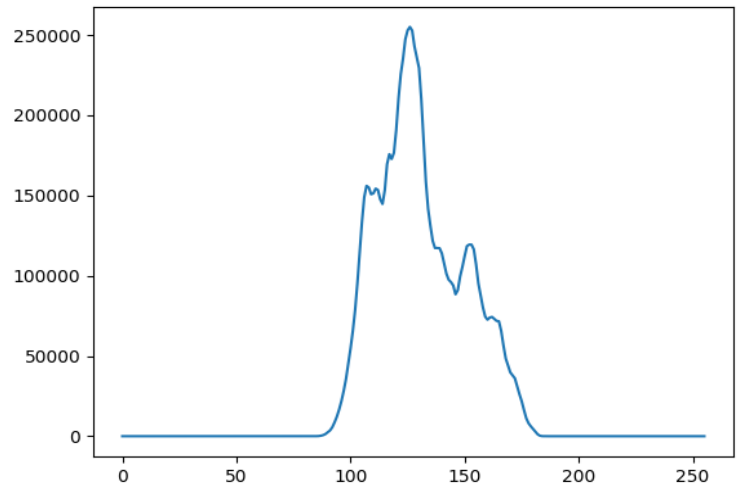

Figure 3. Histogram
Mean:129.8333;median:101<145;skewness0.0512<1.0395;k urtosis: $1.8688<3.1787$; standard

deviation:20.11102246;rma:131.38164219;contrast:0.0275;c orrelation:0.9685; energy:0.3975;homogeneity:0.9863.

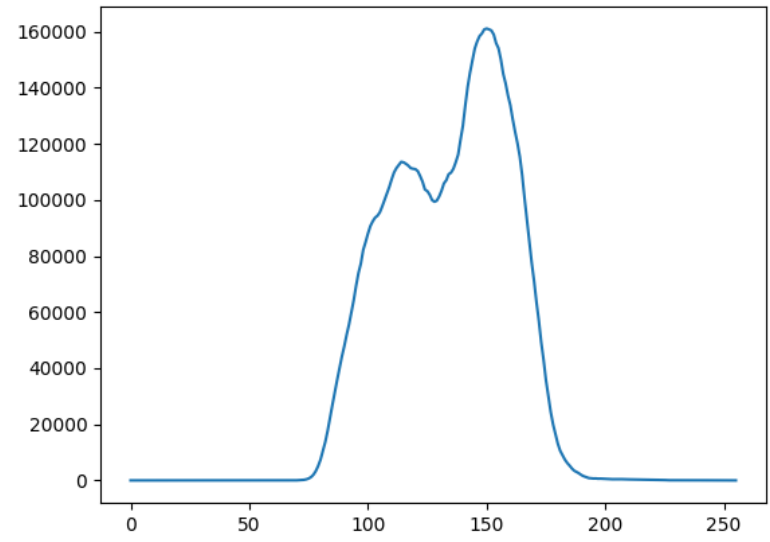

Figure 4.Histogram

Mean:133.8313;median:118<157;skewness:$0.8714<0.7036$;kurtosis:4160;standard deviation:25.52320006;rms:136.24335275;contrast:0.0373;c orrelation:0.9719;energy:0.3147;homogeneity:0.9813

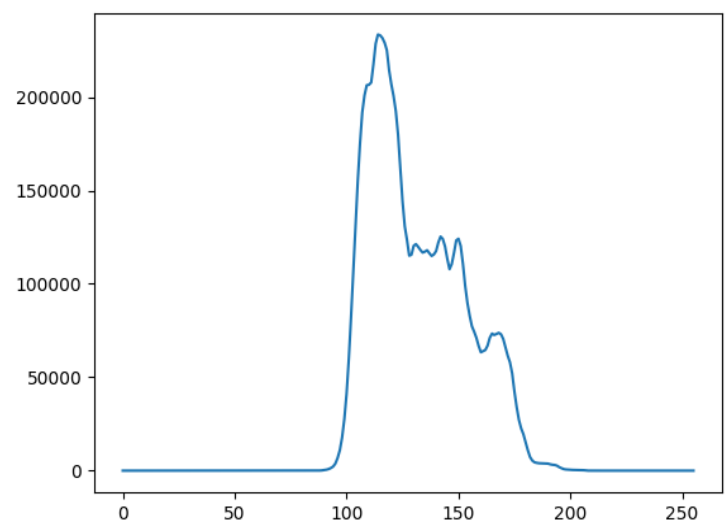

Figure 5.Histogram

Mean:125.1639;Median:92<142;skewness:0.0647<1.5293;K urtosis: $1.4079<4.8508$;standard deviation:31.51423808rms:129.07036026; contrast:0.0421;co rrelation:0.9770; energy:0.2942;homogeneity:0.9789 


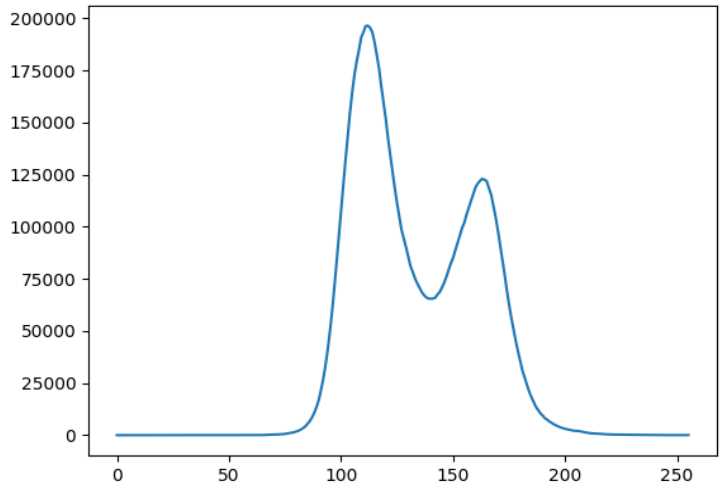

Figure 6.Histogram

Mean:134.9803;median:120<140;skewness:0.0575<1.0605;k urtosis: $1.5166<3.5307 ;$ standard deviation:27.11003464;rms:137.67584433;contrast:0.0734;c orrelation:0.9506; energy:0.3123; homogeneity:0.9636.

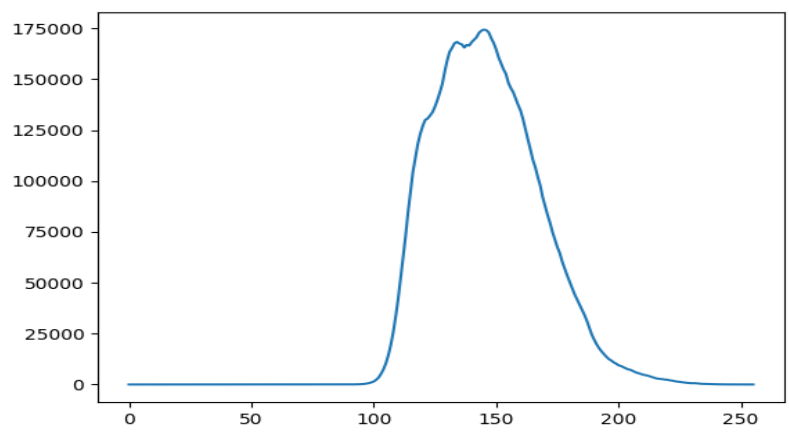

Figure 7.Histogram

Mean:147.1045;median:129<165;skewness:0.1541<1.7123;k urtosis: $2.0448<7.2603$;standard deviation:21.50813091;rms:148.66854198;contrast:0.0605;c orrelation:0.9440;energy:0.3408;homogeneity:0.9698

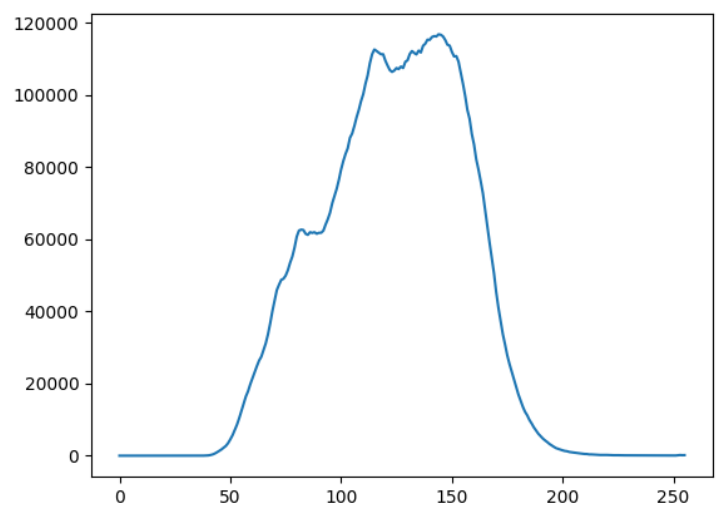

Figure 8.Histogram
Mean:124.3413;median:109<148;skewness:$0.5858<0.9248$; kurtosis: $1.6899<6.8158$; standard deviation: 31.09585432;rms:128.17062677; contrast:0.0451; correlation: 0.9770; energy:0.2561;homogeneity:0.9775

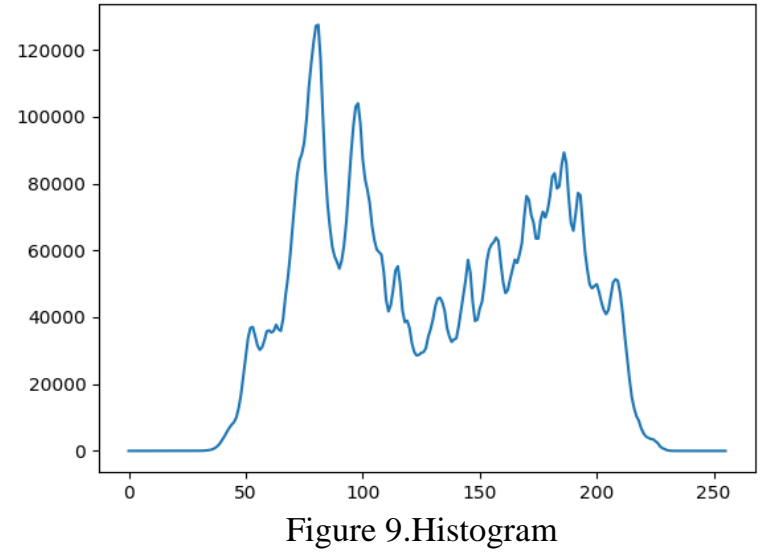

Mean:130.6535;median:94<162;skewness:$0.2379<0.6454$; kurtosis: $1.3015<2.2061$ standard deviation:48.79663407;rms:139.46845158;contrast:0.0219;c orrelation:0.9953; energy:0.1835;homogeneity:0.9890

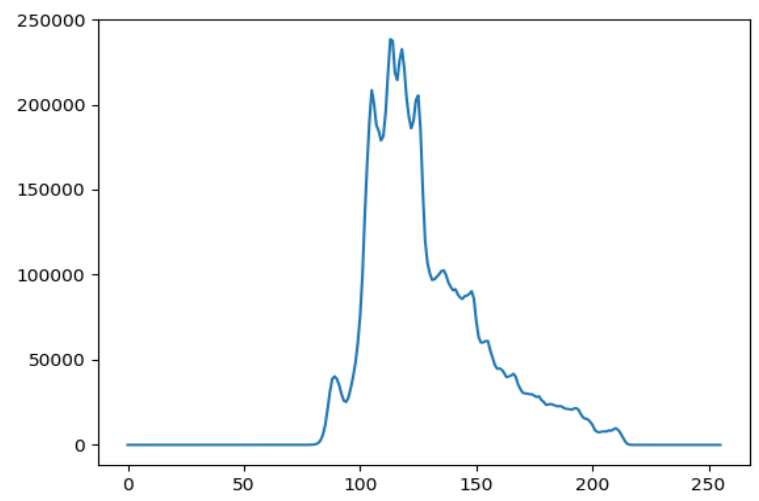

Figure 10.Histogram

Mean:128.2043;median:98<182;skewness$0.1230<1.9574 ;:$ kurtosis:1.4652<6.1033; standard deviation:27.34170277;rms:131.08739927;contrast:0.0183;c orrelation:0.9865; energy:0.3947;homogeneity:0.9908

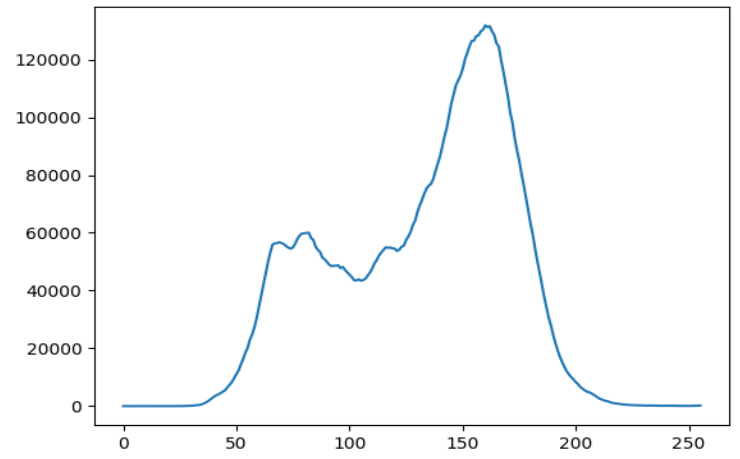

Figure 11.Histogram 
Mean:132.2662;median:74<182;skewness:$0.5488<1.2092 ;$ kurtosis: $1.4529<8.7879$; standard deviation:39.06042822;rms:137.91327273; contrast:0.0581;c orrelation:0.9807;energy:0.2129; homogeneity:0.9709

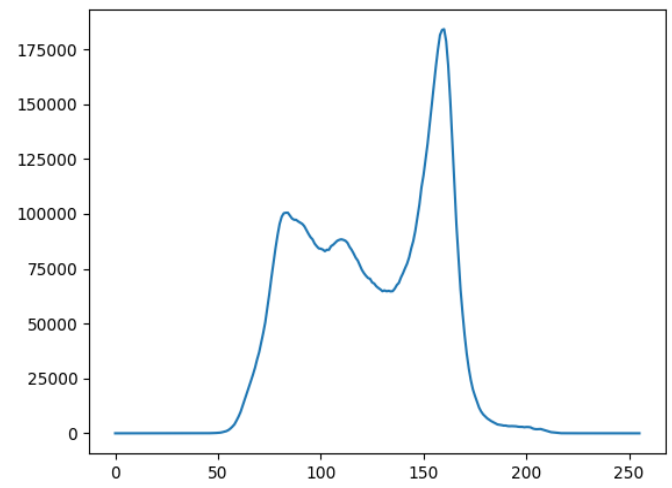

Figure 12.Histogram

Mean:122.7604;median:104<147.50;skewness:$0.9099<0.4662 ;$ kurtosis:1.4229<3.2078; standard deviation:32.44676134;rms:126.97604974;contrast:0.0663;c orrelation:0.9697;energy:0.2290;homogeneity:0.9668.

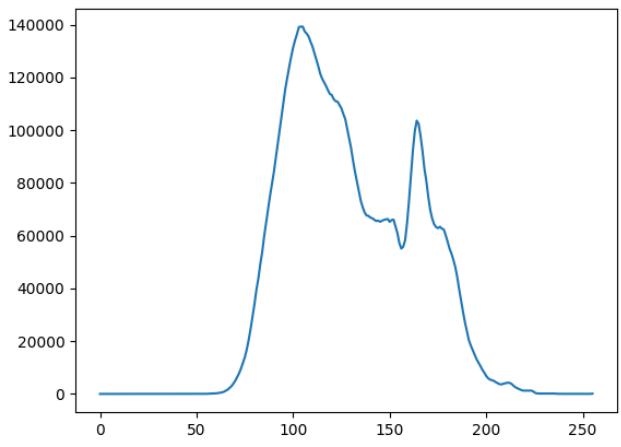

Figure 13.Histogram

Mean:130.7851;Median:114.50<156;skewness:0.0827<0.586 4;kurtosis:1.7463<3.2814;standard deviation:30.27352054;rms:134.24319993;Contrast:0.0489; Correlation:0.9733;Energy:0.2580;Homogeneity:0.9756;

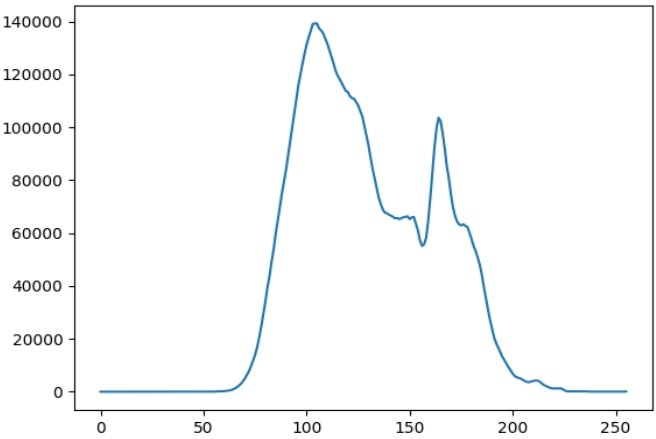

Figure 14.Histogram
Mean:128.1184;median:101<149;skewness:0.0255<0.9811;k urtosis: $1.7288<4.8776$; standard deviation:33.35030;rms:132.38789812; contrast:0.0684;correl ation:0.9675;Energy:0.2454;Homogeneity:0.9659

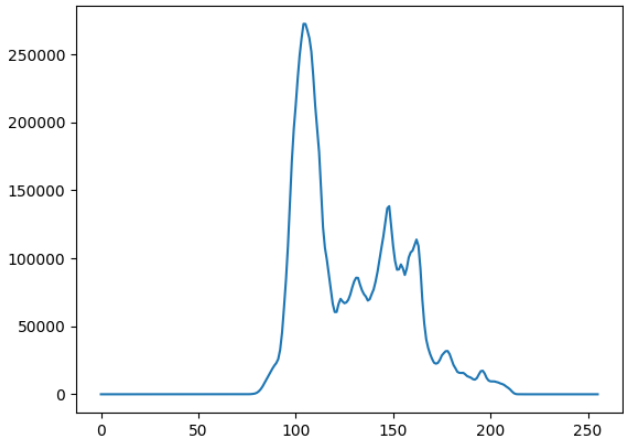

Figure 15.Histogram

Mean:128.0944Median:107<146;skewness:$0.1146<0.8071 ;$ Kurtosis: $1.3600<2.5561$;standard deviation:27.73282497;rms:131.06210081;contrast:0.0263;c orrelation:0.9810;energy:0.3561;homogeneity:0.9868

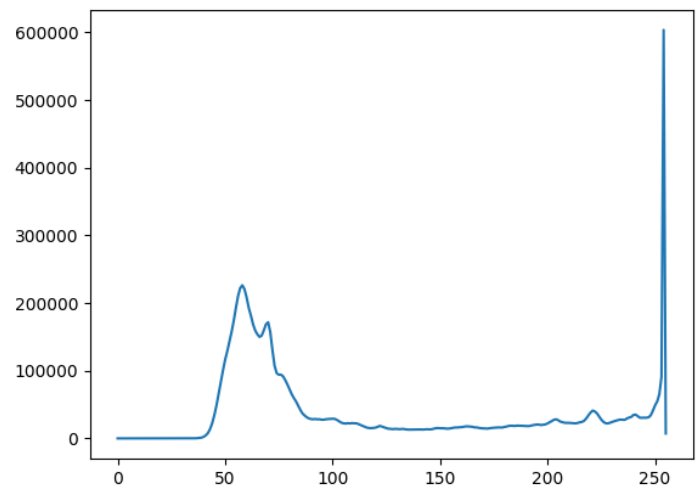

Figure 16 .Histogram

Mean:118.9123;Median:28<254;skewness:$1.7173<1.13570 ;$ Kurtosis: $1.5961<12.6990 ;$ standard deviation:75.81545694;rms:141.02533014;contrast:0.0372;c orrelation:0.9964; energy:0.1964;homogeneity:0.9814.

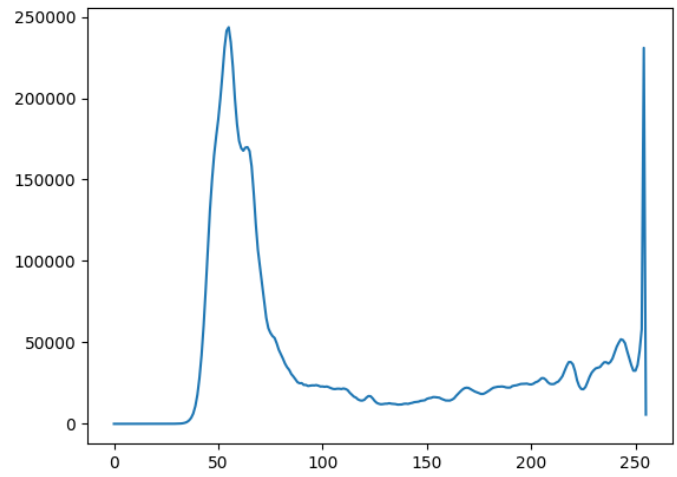

Figure 17.Histogram 
Mean:113.3192;median:33<251;skewness:$1.4322<1.3520 ;$ kurtosis: $1.6723<5.8829$;standard deviation:74.02636905;rms:135.35562802; contrast:0.0365;c orrelation:0.9965;energy:;0.2176homogeneity:0.9818.

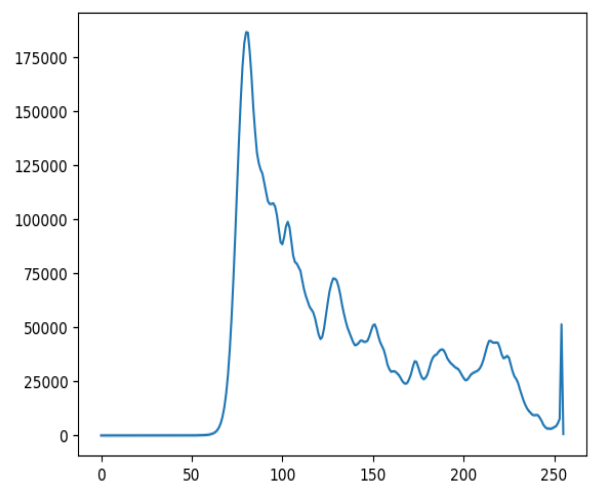

Figure 18.Histogram

Mean:126.9500;median:75<166;skewness:0.2204<0.9564;ku rtosis: $1.5995<2.9402$;standard deviation:51.22084864;rms:136.89371783; contrast:0.0447;c orrelation:0.9906;energy:0.2042;homogeneity:0.9766.

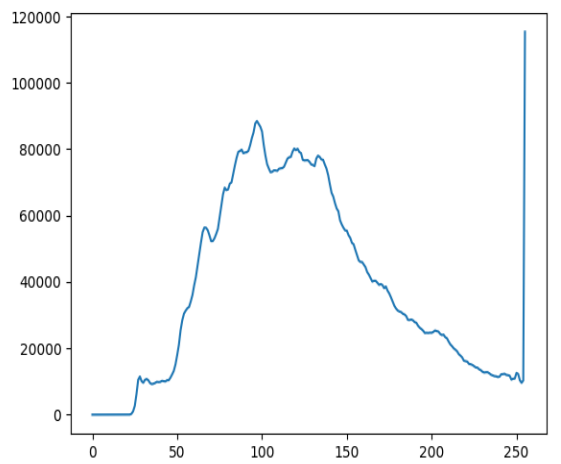

Figure 19.Histogram

Mean:125.9785;median:79<191;skewness:-

$0.5358<1.7931 ;$ kurtosis: $1.5018<6.4023$; standard deviation:51.00315175;rms:135.91138296; contrast:0.0406;c orrelation:0.9917;energy:0.1703;homogeneity:0.9797.

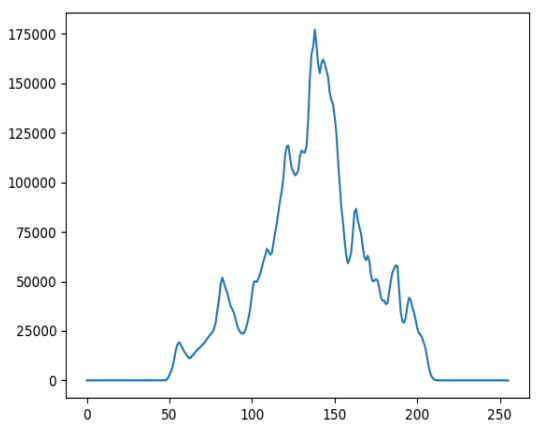

Figure 20.Histogram
Mean:135.8938;median:116<154;skewness:$1.0281<2.5041 ;$ kurtosis: $1.6572<8.4744$ standard deviation:33.09114471rms:139.86475671;contrast:0.0172;co rrelation:0.9922; energy:0.2692;homogeneity:0.9914.

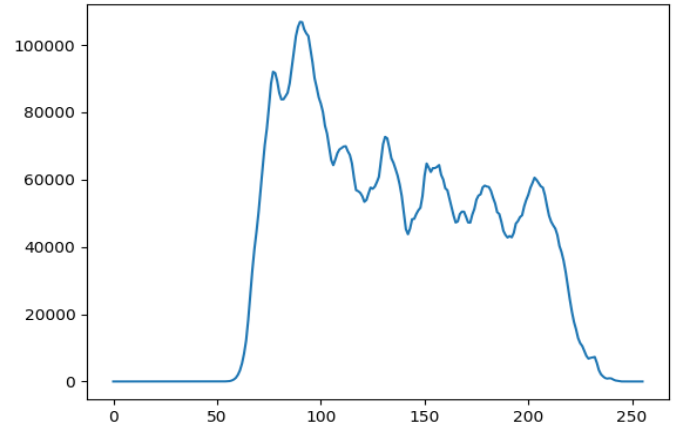

Figure 21.Histogram

Mean:133.1109;median:108<175;skewness:-

$0.4851<0.6954$;kurtosis: $1.4972<2.7456$; standard deviation:46.91947015;rms:141.13805825;contrast:0.0213;c orrelation:0.9948; energy:0.1940;homogeneity:0.9893.

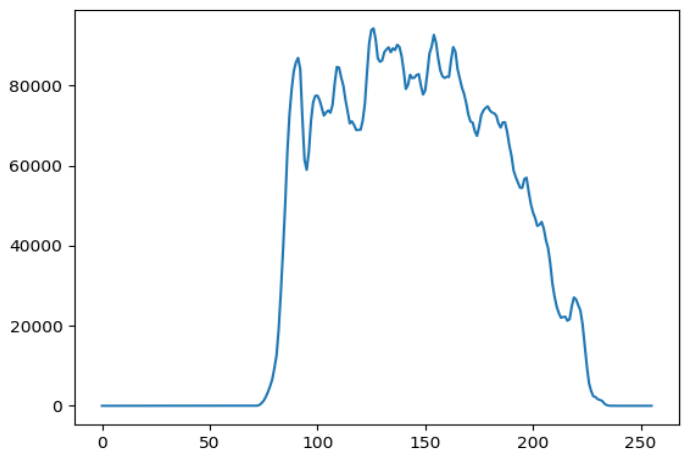

Figure 22.Histogram

Mean:145.1251;median:91<219;skewness:$0.6133<1.2910$; kurtosis: $1.3655<3.9802$;standard deviation:37.86515498;rms:149.98354097;contrast:0.0222;c orrelation:0.9921; energy:0.2168; homogeneity:0.9889.

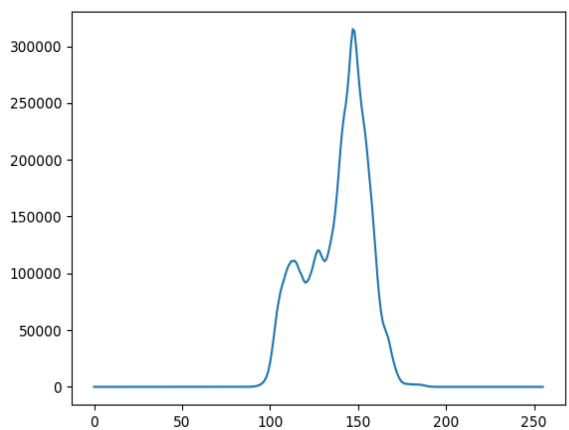

Figure 23.Histogram 
Mean:139.1630;median:121<159.50;skewness:$1.2870<1.8513$; kurtosis: $1.4025<8.5525$; standard deviation: 17.45541587 ;rms:140.25342816; contrast:0.0474;c orrelation:0.9228;energy:0.2168; homogeneity:0.9889.

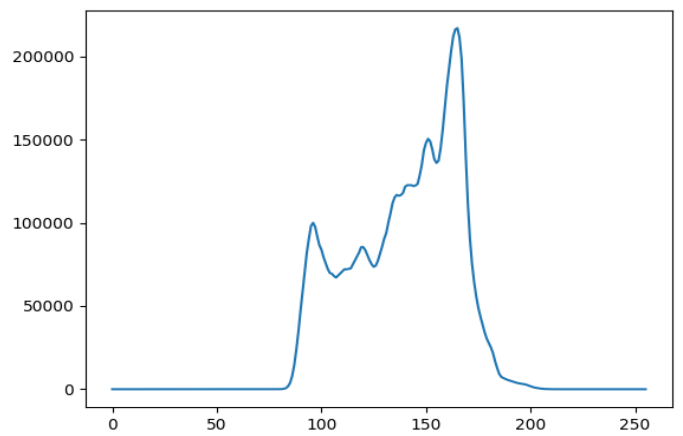

Figure 24.Histogram

Mean:115.6190;median:68<133;skewness:$0.6328<4.1074$;kurtosis:1.6294<23.3862;standard deviation:25.59995128rms:118.41919954;contrast:0.0674;co rrelation:0.9442;energy:0.3167;homogeneity:0.9663

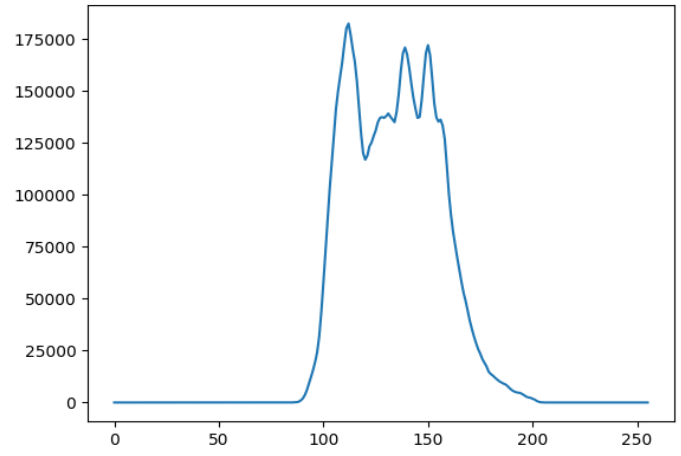

Figure 25.Histogram

Mean:140.3790;median:101<173;skewness:$1.3637<1.206$; kurtosis: $1.4465<4.4072$; standard deviation:26.01683883;rms:142.76951563; contrast:0.0546;c orrelation:0.9640;energy:0.2825;homogeneity:0.9727.

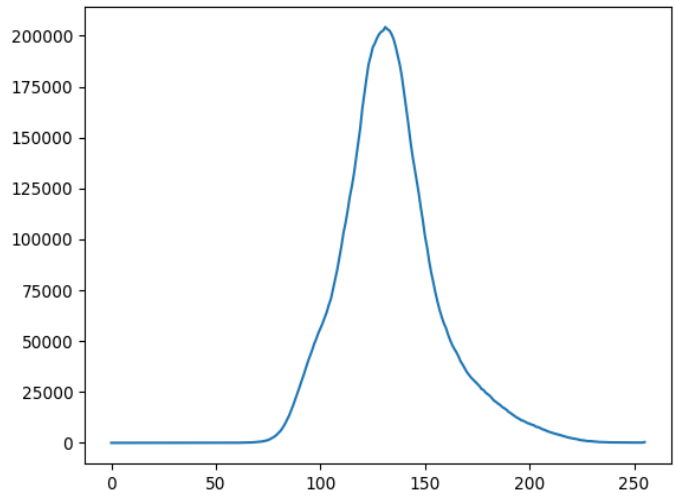

Figure 26.Histogram
Mean:134.3254;median:109<170'skewness:$1.27229<2.1209$; kurtosis:1.8403<12.8192; standard deviation:25.14006955;rms:136.65777274;contrast:0.1091;c orrelation:0.9140;energy:0.3030;homogeneity:0.9465

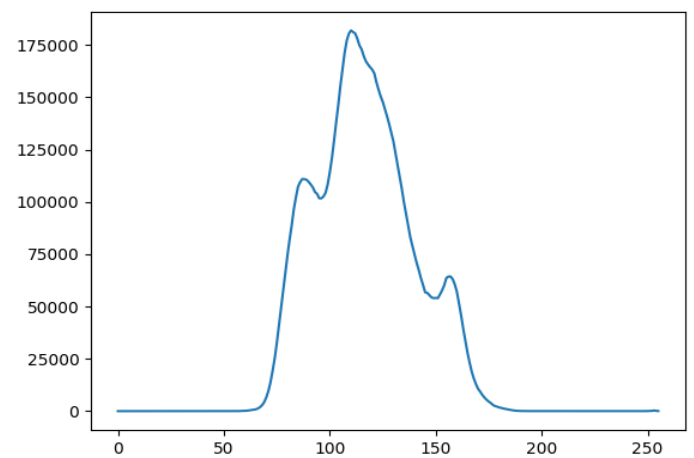

Figure 27.Histogram

Mean:133.4923;median:111<150;skewness:$0.5805<0.9212$; kurtosis: $1.3693<4.0873$; standard deviation:23.85328509;rms:135.60672991;contrast:0.0346;c orrelation:0.9620;energy:0.3805;homogeneity:0.9827

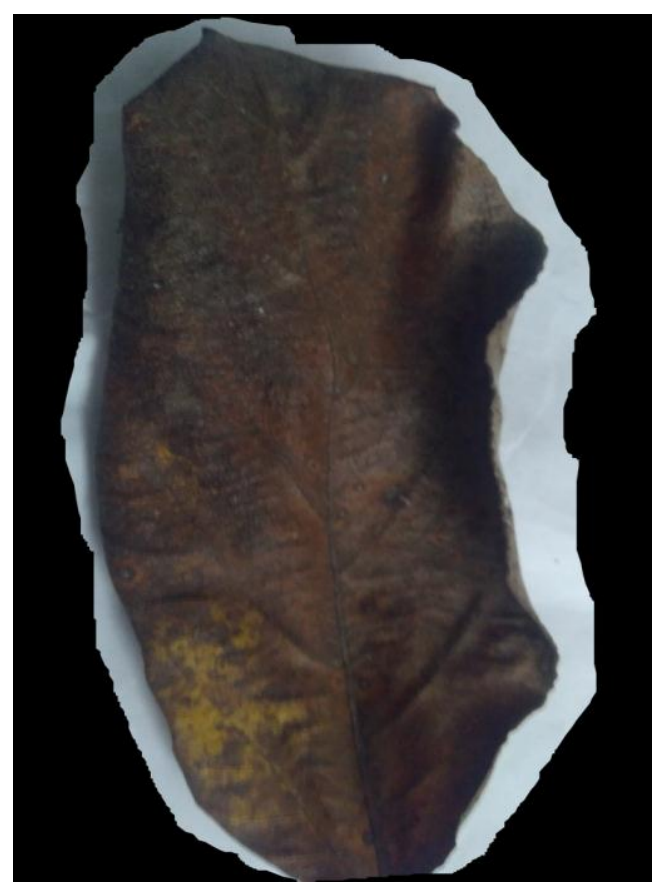

Figure 28.Polluted leaf area calculation

If we Observe the above histograms and statistical values data distribution is either left skewed or symmetry. Mean is always less than median.Skewness values give approximately lesser than 1 based on the above results conclude that polluted leaves data distribution can be find using skewness values.Above results are very useful in 
automatic detection of polluted leaves. Using ImageJ Software areas of leaves were calculated and we conclude that Polluted leaves area much much lesser than normal leaves.

\section{CONCLUSION AND FUTURE SCOPE}

From the above histograms and statistical measures automatic detection of polluted leaves are possible.In future want to implement big-data map-reduce methodology for classification of polluted leaves.

Polluted leaves database available at http://www.sunresearch.yolasite.com

\section{ACKNOWLEDGMENT}

Thanks to Dr .vakulbharanam venkata Krishna for his encouragement to write this article.And My Parents and apple School in mummidivaram,AndhraUniversity college of engineering(Autonomous) Visakhapatnam.

\section{REFERENCES}

[1] Sumitra Giri ,"Effect of air pollution on chlorophyll content of leaves",Current Agriculture Journal Vol,1(2),93-98(2013) http://dx.doi.org/10.12944/CARJ.1.2.04

[2] B.V Ramana Reddy A.Suresh Classification of Textures Based on Features Extracted from Preprocessing Images on Random Windows International Journal of Advanced Science and Technology Volume 9, August, 2009

[3] A New Approach for Texture Segmentation Using Gray Level Textons International Journal of Signal Processing, Image Processing and Pattern Recognition Vol. 6, No. 3, June, 2013.

[4] Detection of unhealthy region of plant leaves and classification of plant leaf diseases using texture features Agric Eng Int: CIGR Journal Open access at http://www.cigrjournal.org Vol.15 No.1 211

[5] Alireza Pourkhabbaz.Nayerah Rastin, "Influence of Environmental Pollution on leaf properties of urban plane trees,Platanus orientalis L.", Bull Environ Contam Toxicol(2010)85:251255,DOI 10.1007/s00128-010-0047-4 Busgenweg 1a,37077 Gottingen,Germany ,Springer 12 December 2009/Accepted:11 june 2010/Published online:25 june 2010.

[6] University "Al.I.Cuza"Iasi , "Air Pollution Effects on the Leaf Structure of some Fabaceae Spacies", Print ISSN 0255965X;Electronic ISSN 1842-4309,Not.Bot.Hort.Agrobot.Cluj 37(2) 2009,57-63,Available online at www.notulaebotanicae.ro

[7] Anett C.Hansen and Harald K.Selte,"Air Pollution and Sick-leaves -is there a connection? A Case study using Air Pollution Data from Oslo",Discussion Papers No-197 Statistics Norway.july1997.

[8] V.Venkata Krishna, "Classification of metals using texture features", International Journal of Computer Science and Technology and Vol.4, Issue.spl-4, ISSN:09768491(online)/ISSN:2229-4333, 2013.
[9] Revathi, P., and M. Hemalatha. "Cotton Leaf Spot Diseases Detection Utilizing Feature Selection with Skew Divergence Method." International Journal of Scientific Engineering and Technology (ISSN: 2277-1581) 3 (2014): 22-30.

[10] Rastogi, A.; Arora, R.; Sharma, S., "Leaf disease detection and grading using computer vision technology \& fuzzy logic," in Signal Processing and Integrated Networks (SPIN), 2015 2nd International Conference on , vol., no., pp.500-505, 19-20 Feb. 2015.

[11] Classification of metals using texture features ,suneel kumar badugu, venkata Krishna vakulabharanam et all IJCST IJCST Vol. 4, Iss ue Spl - 4, Oct - Dec 2013, ISSN : 0976-8491 (Online) | ISSN : 2229-4333 (Print).

[12] Comparative study of various classification algorithms combined with $\mathrm{K}$ means algorithm for Leaf Identification International Journal Of Engineering And Computer Science ISSN: 2319-7242 Volume 5 Issues 6 June 2016, Page No. 17022-17025.

[13] Abdul Kadir et all Leaf classification using shape,color,and texture features, International Journal of Computer Trends and Technology-July to Aug Issue 2011, ISSN: 2231-2803.

[14] T. Acharya, \& A.K. Ray, "Image Processingg Principles and Applications", New Jersey: John Wiley \& Sons, Inc, 2005.

[15] Y.A.O. Min, Y.I. Win-Sheng,S. Bin, \&D.A.I. Hong-Hua, "An Image Retrieval System Based on Fractal Dimension", Journal Zheijang University Science, vol. 4(4), p. 421-425, 2003. M. Petrou, \& P.G. Sevilla, "Image Processing Dealing with Texture", Chichester: John Wiley \& Sons, Ltd., 2006.

[16] M. Petrou, \& P.G. Sevilla, "Image Processing Dealing with Texture", Chichester: John Wiley \& Sons, Ltd., 2006.

[17] A. Gebejes, \& Huertas, R. (2013). Texture Characterization Based on Grey-Level Co-Occurrence Matrix.

[18] Studying the Kidney Textural Using Statistical Features and Local Binary Pattern Vol.20 (4), December, 2017, pp.64-76 Journal of Al-Nahrain University.

\section{Authors Profile}

suneel kumar badugu research scholar, doing research from Andhra university college of engineering(autonomous) in computer science and systems engineering department,under th esteemed guidance of Dr K .Raja kumar.worked as an assistant professor in CHAITANYA INSTITUTE OF ENGINEERING AND TECHNOLOGY ,Rajahmundry during 2011-2013.And also worked as an assistant professor in BONAM VENKATACHALAMAIAH INSTITUTE OF TECHNOLOGY AND SCIENCE BATLAPALEM.Now working as a teacher in apple school at mummidivaram of east Godavari district of Andhra Pradesh.Published 5 articles in different journals.Completed M.Tech in information technology from Andhra university college of engineering(autonomous).Completed B.Tech from SAGI RAMAKRISHNAMA RAJU ENGINEERING COLLEGE.Completed Intermediate from SRI KONASEEMA BANOJI RAMARS COLLEGE AMALAPURAM.schooling from ZILLA PARISHAD HIGH SCHOOL RAJAVOMMANGI.Attended so many workshops . 
Dr K Raja Kumar As an Assistant Professor in Andhra University College Of Engineering (Autonomous) in Visakhapatnam. Dr.K Raja Kumar Completed his B.E(E.C.E) In 1998 from Andhra University College Of Engineering(Autonomous)

Visakhapatnam. And Completed his M.Tech(CST) from Andhra University in 2000.And Received Doctorate From PROFESSOR EMERITUS Prof. P.Seetharamaiah Recipient Of Best Researcher Award from Andhra

University, India (1998) Member of Visiting Staff under Academic Link Exchange Scheme sponsored by British Council-UK and University Grants Commission-India, 1993,Andhra University College Of Engineering ,Department Of Computer Science And Systems Engineering .Dr K.RajaKumar Having two years Research Experience from Research Center-IMART DRDO Hyderabad. And having Thirteen Years Teaching and Research Experience from Various Institutes. And He Published Different Journals and Conducted Conferences, Workshops and Attended different Workshops.

Dr Venkata Krishna Vakula Bharanam Professor and principal in Nishita college of engineering and technology in Hyderabad.Worked at different reputed engineering colleges namely chaitany abharati institute of technology ,Hyderabad,anurag college of engineering, Hyderabad and Godavari institute of engineering and

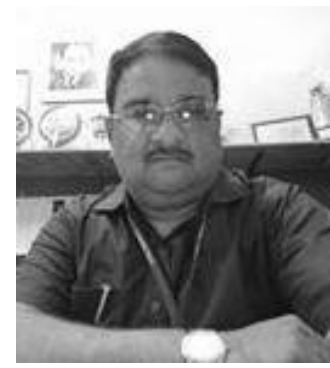
technology Rajahmundry. And published many articles in reputed journals and attended many conferences and workshops.And conducted many workshops.And occupied different positions namely principal ,academic coordinator,board of studies member etc.Professor having 30 years experience in research and teaching as well.

Dr B.Prajna is an associate Professor in the department of computer Science and Systems Engineering ,Andhra University College of Engineering (Autonomous).Dr Prajna area of specialization is Data Mining. Professor Prajna Published many research articles in Reputed national and International journals.And also Dr Prajna Conducted conferences. Dr Prajna

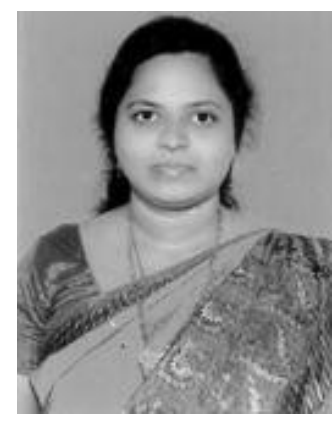
has 15 years teaching as well as research Experience. 